\title{
Capacity for microtubule reorganization and cell wall synthesis in cytoplasts of the green alga Mougeotia
}

\author{
Moira E. Galway**, G. J. Hyde ${ }^{+}$, and Adrienne R. Hardham* \\ Plant Cell Biology Group, Research School of Biological Sciences, The Australian National University, Canberra, ACT
}

Received September 1, 1993

Accepted November 8, 1993

\begin{abstract}
Summary. A small proportion of nucleate subprotoplasts (karyoplasts) and enucleate subprotoplasts (cytoplasts) are formed during the preparation of protoplasts from the filamentous green alga $\mathrm{Mou}$ geotia. Regeneration of Mougeotia protoplasts is an orderly process known to involve reorganisation of cortical microtubules into polar arrays centered upon two opposing foci, synthesis of new cell walls and elongation to reform cylindrical cells. The ability of cytoplasts to carry out microtubule reorganisation and cell wall synihesis was investigated by combining Hoechst staining, to distinguish cytoplasts from karyoplasts and protoplasts, with immunofluorescent staining of microtubules and Calcofluor or Tinopal staining of cell walls. Cytoplasts survived at least $20 \mathrm{~h}$ in culture, but did not elongate. However, cytoplasts did participate in the first steps of protoplast regeneration. The majority of cytoplasts synthesized some cell wall material, while a small proportion was able to form ordered arrays of cortical microtubules indistinguishable from those in regenerating nucleate protoplasts. These results demonstrate the ability of plant microtubules to form new, orderly arrays in the absence of a nucleus, and suggest that the reestablishment of axiality in the protoplasts does not require a nucleus or nuclear DNA transcription.
\end{abstract}

Keywords: Alga; Cell wall; Cytoplast; Microtubule; Mougeotia; Protoplast.

Abbreviations: MT microtubule; MAP microtubule associated protein.

\section{Introduction}

The generation and analysis of enucleated cells and hybrid cells formed by centrifugation, microsurgery, or

\footnotetext{
* Correspondence and reprints: Plant Cell Biology Group, Research School of Biological Sciences, The Australian National University, GPO Box 475, Canberra, ACT 2601, Australia.

** Current address: Department of Biology, The University of Michigan, Ann Arbor, Michigan, U.S.A.

+ Current address: Department of Biology, York University, North York, Ontario, Canada.
}

fusion of an enucleate cell with a different nucleus or a nucleated cell have provided valuable information on the effects of different combinations of nuclear and cytoplasmic genes, nuclear-cytoplasmic relationships, and the respective roles of nucleus and cytoplasm in cellular growth and development (Bunn 1982, Reinert and Binding 1986). Detailed studies of enucleated cells of the marine green alga Acetabularia have revealed, for example, the persistence of circadian rhythms and the ability to continue normal processes of growth and morphogenesis for weeks in the absence of a nucleus (Schweiger and Berger 1979). Enucleated Acetabularia cells have unusually stable RNA messages coding for all proteins required during growth and development, and these are present in the cytoplasm before they are required. However, complex cellular processes may still proceed or even be initiated in other enucleate cells in which mRNAs are expected to have more typical lifetimes (minutes to hours, Raghow 1987). One process is the rearrangement of initially unordered cytoskeletal elements into highly ordered arrays that are usually similar to those observed in the cytoplast source cells. This process has been most often studied in animal cytoplasts (Karsenti et al. 1984, Gelfand et al. 1985, Bershadsky and Vasiliev 1988, Guha Roy and Bhisey 1992, and references therein), but investigation of two types of plant cytoplasts suggests that the plant cell cytoskeleton has an intrinsic capacity for self-organization (Haemanthus endosperm: Bajer and Molè-Bajer 1982, 1986; pollen cytoplasts: Rutten and Derksen 1990, 1992). To better understand the "autonomous" properties of the plant cytoskeleton we focused on the 
ability of cytoplasts of the filamentous green alga $\mathrm{Mou}$ geotia to participate in the rapid, highly ordered process of protoplast regeneration that is characteristic of this species.

Mougeotia protoplasts have the ability to regenerate their cell walls, elongate and divide to give rise to new filaments (Marchant and Fowke 1977). Cortical MTs are transversely aligned in the cylindrical algal cells, but become unordered during protoplast formation (Galway and Hardham 1986). Three to four hours later, the cortical MTs form new arrays in which MTs are centered upon two foci located on opposite sides of each cell. Polar arrays of this type do not occur in intact filament cells. Subsequently, a circumferential band of closely aligned MTs forms which intersects the two cortical MT foci. New cell wall deposition can be detected by Calcofluor or Tinopal staining about $2 \mathrm{~h}$ after protoplast isolation (Marchant and Hines 1979, Galway and Hardham 1986). Cell wall microfibrils are initially unordered but subsequent oriented deposition in a band overlying the band of MTs leads to cell elongation (Galway and Hardham 1986). Thus as in many other plant cells, development and maintenance of an axis of elongation in Mougeotia is dependent on the ordered deposition of cellulose microfibrils in the cell wall which in turn is regulated by cortical MTs within the cells (Marchant 1979, Gunning and Hardham 1982).

Enucleate cytoplasts also form during the preparation of protoplasts from Mougeotia filament cells. The DNA-staining fluorochrome Hoechst 33662 permits enucleate cytoplasts to be distinguished unambiguously from intact protoplasts and nucleate karyoplasts and enabled us to investigate the ability of cytoplasts to carry out the two critical processes of protoplast regeneration in Mougeotia, i.e., cortical MT reorganisation and cell wall synthesis.

\section{Materials and methods}

Mougeotia sp. collected from the National Botanic Gardens, Canberra, Australia was cultured as previously described (Galway and Hardham 1986). Protoplast isolation, fixation and the staining of MTs by indirect immunofluorescence microscopy was also carried out as described by Galway and Hardham (1986). Briefly, protoplasts released by enzymic digestion of the cell walls of Mougeotia filaments were collected by filtration and centrifugation. Protoplast age was calcuated as the time elapsed from the addition of the first enzymefree plasmolysis medium to newly isolated protoplasts. At various times after protoplast isolation samples were prepared for immunofluorescence microscopy. Protoplasts were attached to polylysinecoated slides and fixed in $1 \% \mathrm{v} / \mathrm{v}$ glutaraldehyde, followed by extraction in $2 \%$ Triton $X-100$, and then by two washes in $0.1 \%$ $\mathrm{NaBH}_{4}$. They were then incubated in a monoclonal anti- $\beta$-tubulin (Cat. no. M.357, Amersham, Sydney, Australia) followed by staining with a fluorescein-conjugated secondary antibody (Silenus, Dandenong, Vic., Australia). Subsequently nucleate and enucleate protoplasts were easily distinguished after staining in a $20 \mathrm{mg} / \mathrm{ml}$ solution of the DNA-binding fluorochrome Hoechst 33662 (Hoechst-Roussel, NJ, U.S.A.) in phosphate-buffered saline for $10 \mathrm{~min}$ before rinsing in distilled water and mounting in Moviol mounting medium (Osborn and Weber 1982) containing 2\% n-propyl gallate (Sigma Chemical Co., St. Louis, MO, U.S.A.)

In two separate experiments, $21 \mathrm{~h}$ old protoplasts were stained for $15-20 \mathrm{~min}$ in the dark with a $0.01-0.1 \%$ solution of the fluorescent cell wall stain Tinopal LPW which as a stilbene-based fluorescent brightener stains $\beta$-linked glucans including cellulose (Maeda and Ishida 1967, Hughes and McCully 1975, Itoh et al. 1986), before fixation and processing for immunofuorescence microscopy (for a

Figs. 1-7. Light or fluorescence micrographs of plasmolysed cell (Fig. 1), protoplasts (Figs. 2 and 6) and cytoplasts (Figs. 3-5 and 7) of Mougeotia. Bars: Fig. 1, $20 \mu \mathrm{m}$; in Fig. 2 for Figs. 2-7, $20 \mu \mathrm{m}$

Fig. 1. Spontaneous cleavage of a plasmolyzed cell during protoplast preparation has produced two subprotoplasts, one nucleate and one enucleate (stars), to be released by further enzymic degradation of the cell wall. Note protoplast just emerging from open end of adjacent cell (arrowhead)

Fig. 2. In a typical ordered MT array in a $6 \mathrm{~h}$ old regenerating protoplast aligned cortical MTs extend between two opposing cortical foci (F)

Fig. 3. Ordered MTs extend between two opposed cortical MT foci, viewed a on the upper surface and $\mathbf{b}$ on the lower surface of a $5 \mathrm{~h}$ old cytoplast. $\mathrm{c}$ Hoechst stains cytoplasmic DNA but reveals no nucleus

Fig. 4. An ordered cortical MT array viewed in the plane of a the upper surface and $\mathbf{b}$ lower surface (revealing a bipartite focus) of a $22 \mathrm{~h}$ old cytoplast. $\mathrm{c}$ In optical cross-section Hoechst and Tinopal staining reveals a cell wall has formed, but no nucleus is present

Fig. 5. a Upper surface view of MTs centered on one cortical focus of a 21 h old cytoplast. MT-free area $(X)$ suffered damage during fixation. b In optical cross-section, Hocchst and Tinopal staining reveals very faint cell wall and no nucleus

Fig. 6. a Upper surface view of cortical MTs intersecting at a complex cortical MT focus $(F)$ of a $22 \mathrm{~h}$ old protoplast. Not all MTs are in focus due to deformation of protoplast surface during processing. b In optical cross-section, Hoechst and Tinopal stain the nucleus ( $N$ ) and well-developed cell wall, respectively

Fig. 7. a Upper surface view of $6 \mathrm{~h}$ old cytoplast with unorganised cortical MTs exhibiting localized areas of alignment and intersection. b Combined Hoechst and Tinopal staining reveals no nucleus or cell wall in optical cross-section of cytoplast 

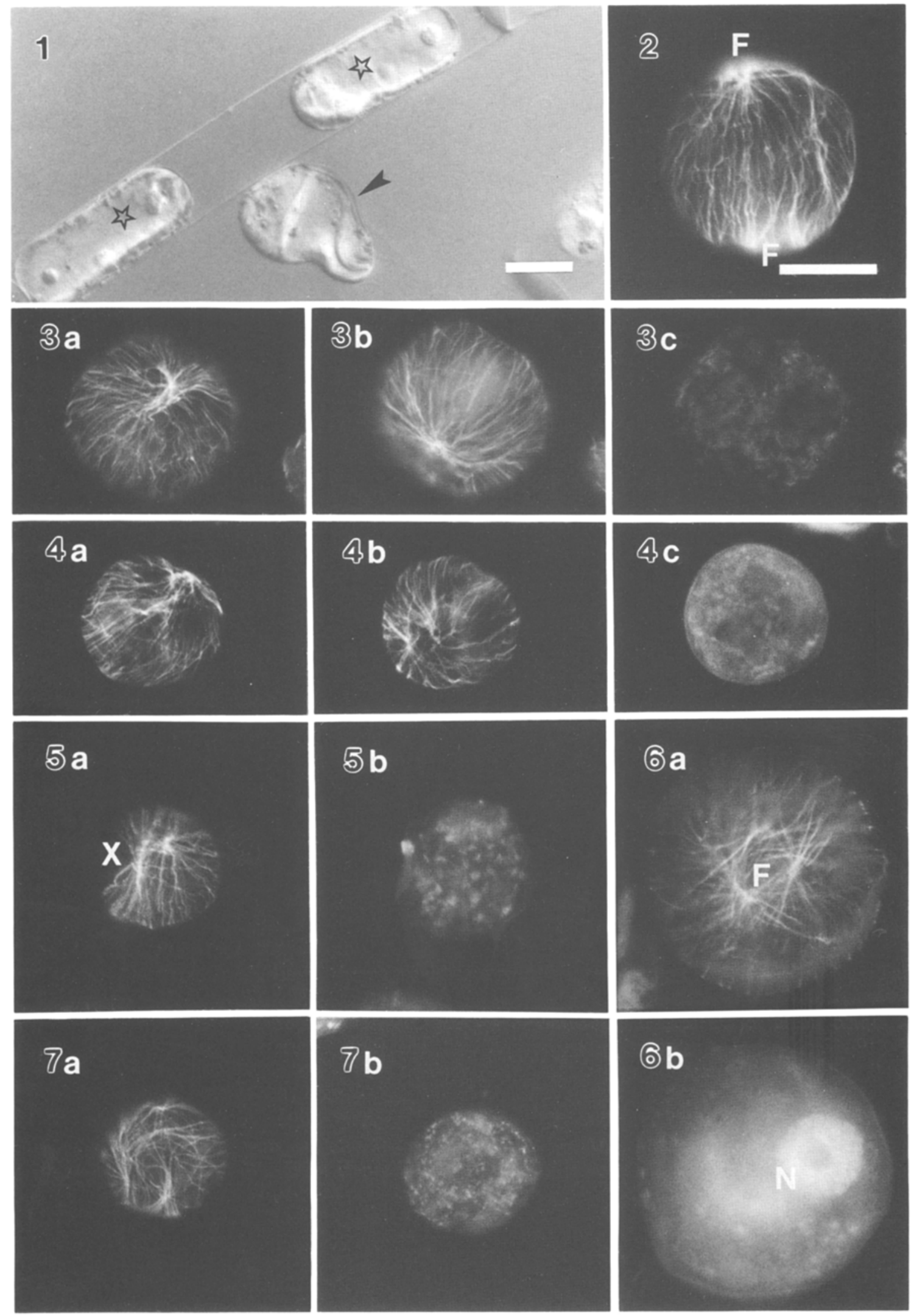
detailed discussion of the use of Tinopal LPW as a cell wall stain, see Galway and Hardham 1986). Immunofluorescently stained protoplasts were examined using either a Zeiss Photomicroscope or Universal Research Microscope, each equipped with appropriate filter sets, HBO 200 mercury burners and epifluorescence optics. Fluorescence micrographs were recorded on Kodak Tri-X film. All quantitative results presented here were drawn from samples of $200-$ 550 protoplasts.

\section{Results}

Incubation of Mougeotia filaments in the plasmolysing enzyme solution caused some protoplasts to spontaneously cleave into two portions before their release by enzymic digestion of the cell walls. Cleavage yielded two types of subprotoplasts: nucleate (karyoplasts) and enucleate (cytoplasts) (Fig. 1). Cleavage of protoplasts into more than two portions was not observed. Later, intact protoplasts and subprotoplasts emerged through openings in the enzyme-weakened cell walls, particularly at the cross walls which often became detached from the remainder of the cell walls. Newly isolated protoplasts had no detectable cell walls, and did not fluoresce when stained with Calcofluor or Tinopal.

Hoechst staining of nuclear DNA in glutaraldehydefixed protoplasts enabled cytoplasts to be reliably distinguished from protoplasts and karyoplasts (Figs. 37). Cytoplast diameter was $10-50 \%$ less than the average protoplast diameter of $35 \mu \mathrm{m}$ (compare Figs. 2 and 6 to Figs. 3-5 and 7). The proportion of cytoplasts in protoplast populations sampled at different ages varied from 10 to $23 \%$, but did not significantly decrease over the first 21 h of protoplast culture (Table 1).

Cortical MTs were unordered in newly-formed protoplasts and subprotoplasts of Mougeotia (not shown; see Galway and Hardham 1986), but within $2 \mathrm{~h}$ after protoplast isolation ordered, polar arrays of MTs were

Table 1. Proportion of cytoplasts among regenerating protoplasts of different ages

\begin{tabular}{ll}
\hline Age of protoplasts $(\mathrm{h})$ & \% cytoplasts \\
\hline 2 & $13.4 \pm 2.3$ \\
3 & $17.6 \pm 3.8$ \\
4 & $19.3 \pm 4.2$ \\
6 & $13.2^{\mathrm{b}}$ \\
21 & $13.0 \pm 3.7$
\end{tabular}

2 Values indicate the mean $\%$ cytoplasts \pm standard deviation obtained from same age samples of $2-6$ different protoplast preparations

b $\%$ cytoplasts calculated from sample of one $6 \mathrm{~h}$ old protoplast preparation formed in an average of $19 \%$ of nucleate protoplasts (including karyoplasts) and 5\% of cytoplasts (Fig. 8). The number of these polar MT arrays in protoplasts and subprotoplasts increased rapidly, and by $3 \mathrm{~h}$ after isolation an average of $66 \%$ of nucleate protoplasts and $13 \%$ of cytoplasts possessed the ordered arrays. After $3 \mathrm{~h}$ there was no further significant increase in the proportion of cytoplasts with ordered MTs, although the proportion of nucleate protoplasts with ordered MTs continued to increase (Fig. 8).

The polar MT arrays formed in cytoplasts were indistinguishable from those typically formed in nucleate protoplasts (compare Figs. 2 and 6 a to Figs. 3 a , b, 4 a, $\mathrm{b}$, and $5 \mathrm{a}$ ). The foci of $21-22 \mathrm{~h}$ old protoplasts and some cytoplasts have a more complex morphology than younger protoplasts (Figs. $4 \mathrm{~b}$ and 6 ). However, unlike nucleate protoplasts, no formation of MT bands or initiation of elongation was detected in the 21 or $22 \mathrm{~h}$ old cytoplasts. Those cytoplasts which did not develop polar arrays of cortical MTs still possessed intact cortical MT arrays which were often characterized by points of MT intersection and localized areas of MT coalignment (Fig. 7).

Cell wall production in cytoplasts was examined after 21 or $22 \mathrm{~h}$ in culture, as our previous study showed that by this time the majority of nucleate protoplasts have well-developed cell walls that are easily detected by fluorescent cell wall stains after fixation and extraction for immunofluorescence staining of MTs (Galway and Hardham 1986). After $22 \mathrm{~h}$ in culture the

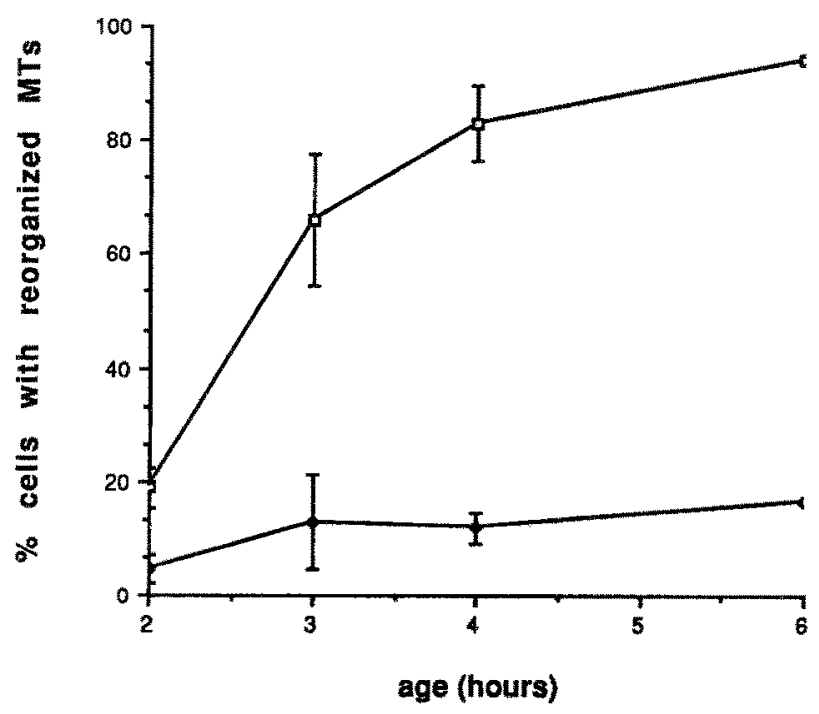

Fig. 8. Time course of cortical MT organisation in protoplasts ( $\square$ ) and cytoplasts $(*)$ during the first $6 \mathrm{~h}$ after isolation. Error bars indicate the standard deviation of each mean 
Table 2. Proportion of 21 h old protoplasts and cytoplasts possessing cell walls detectable by Tinopal staining

\begin{tabular}{lll}
\hline Experiment & \% walled protoplasts & $\%$ walled cytoplasts \\
\hline 1 & $89.8(352)$ & $81.5(53)$ \\
2 & $86.3(408)$ & $88.1(41)$ \\
\hline
\end{tabular}

In brackets, protoplast and cytoplast sample size, respectively

majority of cytoplasts, including those with polar MT arrays, also had cell walls detectable by fluorescent staining with Tinopal (Table 2; Figs. $4 \mathrm{c}$ and 5 b). However, cytoplast cell-wall fluorescence was faint compared to that of nucleate protoplasts (compare Figs. $4 \mathrm{c}$ and $5 b$ to Fig. $6 b$ ).

\section{Discussion}

The results show that some cytoplasts of Mougeotia were capable of developing two features that have previously been reported in regenerating nucleate protoplasts of this species: (1) a highly organised array of cortical microtubules extending between two foci located at opposite sides of the cells and (2) a cell wall.

\section{Reorganization of MTs in cytoplasts}

Cortical MTs in cytoplasts reorganised in one of two ways, either in a highly ordered, pole-to-pole fashion or as unordered arrays with regions of coaligned and intersecting MTs. Formation of polar arrays is the initial step in a precise sequence of cytoskeletal rearrangements by which spherical nucleate protoplasts revert to a normal cylindrical form (Galway and Hardham 1986). Although protoplasts do not form under natural conditions, this regeneration response of $\mathrm{Mou}^{-}$ geotia protoplasts may be important for recovery from wounding or perhaps for development of the ovoid aplanospores or zygospores from contracted cytoplasm within filament cell walls (Kennedy and Hoshaw 1978). Since the polar arrays do not resemble any arrays formed in filament cells, they must form in response to a novel combination of the factors that normally determine MT organization. An investigation of the effects of externally applied stimuli did not detect any effect of light or gravity on the development of protoplasts axiality in Mougeotia, but did implicate mechanical stretching of the plasma membrane as one factor which could influence protoplast axiality indirectly through its effect on MT alignment (White et al. 1990). Williamson (1991) has suggested that MTs responding to small asymmetries in cellular mechanical stresses could magnify these stresses and generate orderly arrays by a positive feedback mechanism. Recent reviews have focussed on the importance of interactions, including mechanical forces, between the cytoskeleton, plasma membrane, and extracellular matrix in influencing the form of cytoskeletal arrays in cells (see Burridge et al. 1988; Roberts 1989, 1990; Ingber 1993).

To implement MT reorganisation into polar arrays must require numerous factors including proteins such as MAPs that are involved in modulating MT biochemistry, and in forming intercytoskeletal and membrane links (Cyr 1991). As in Acetabularia (Schweiger and Berger 1979), long-lived mRNAs may be involved in MT reorganisation in Mougeotia cytoplasts. The limited number of cytoplast-formed polar arrays, and the inability of cytoplast regeneration to progress beyond formation of these arrays, could reflect (1) the metabolic complexity of MT reorganisation, (2) the low probability of cytoplasts inheriting sufficient mRNAs and other metabolic machinery from the source filament cells, and (3) the inevitable breakdown of cellular metabolism in the absence of nuclear gene transcription.

Interestingly, Rutten and Derksen (1990) have described polar arrays similar to those in Mougeotia, but formed by actin microfilaments, in regenerating cytoplasts (and karyoplasts) isolated from pollen tubes of Nicotiana. The close resemblance between the polar MT and actin arrays in these two cell types is even more striking given the fact that the cytoplasts behave in very different ways: Mougeotia cytoplasts do not elongate, while pollen tube cytoplasts exhibit outgrowth and budding (Rutten and Derksen 1990). Also, while formation of a polar MT array has an essential function in the regeneration of Mougeotia protoplasts, pollen tube karyoplasts or cytoplasts do not regenerate to normal pollen tubes. The formation of similar polar arrays suggests that both types of cytoskeletal element are responding to similar organising factors in initially apolar cytoplasts and protoplasts. It may be that the formation of polar cytoskeletal arrays is fundamental to the development of axiality or polarity in spherical plant cells.

Finally, self-assembling properties of MTs may be responsible for local MT alignment in Mougeotia cytoplasts that did not develop polar arrays. Coaligned and crosslinked MTs are characteristic of plant MTs selfassembled in vitro (Chang-Jie et al. 1992). In regenerating pollen tube cytoplasts MTs were also aligned into thick curving or looping strands (Rutten and Derk- 
sen 1992) and electron microscopy revealed extensive crosslinks between these MTs. Williamson (1991) has discussed how MT crosslinking could contribute to MT coalignment. The arrangement of cortical MTs into locally aligned arrays seems to be a process distinct from the formation of polar MT arrays since locally aligned arrays are not formed as part of the normal sequence of MT reorganization in regenerating nucleate protoplasts (Galway and Hardham 1986). Although locally aligned arrays of MTs similar to those seen in the present study form in nucleate Mougeotia protoplasts recovering from drug-induced MT disassembly, these are rapidly replaced by normal polar MT arrays (Galway and Hardham 1989). Mougeotia cytoplasts which do form locally aligned arrays of MTs may not be metabolically capable of forming complex polar arrays.

\section{Cell wall synthesis}

The majority of Mougeotia cytoplasts, like nucleate protoplasts, produced Tinopal-stainable cell walls by 21-22 h after isolation. The weak fluorescence of the cytoplast walls after staining indicates that cytoplasts have probably only a limited capacity for cell wall synthesis, perhaps restricted to a short period after cytoplast isolation before the depletion of protein and carbohydrate reserves inherited from the source cell.

Cell wall synthesis has been seldom reported in other types of cytoplasts. Cytoplasts prepared from calluscultured African violet deposited a thin layer of Tinopal-stainable surface material after several weeks of culture in auxin-supplemented B 5 medium (Bilkey et al. 1982). Up to $10 \%$ of cytoplasts from the yeast Saccharomyces cerevisiae synthesized some $\beta-1,3-$ linked glucan microfibrils in a glucose-containing culture medium supplemented with acetate to overcome a defect in glucose metabolism (Kopecká et al. 1987). More recently, a series of studies has been published on the properties of subprotoplasts prepared from pollen tubes of Nicotiana tabacum (Kroh and Knuiman 1988; Rutten and Derksen 1990, 1992; Rutten et al. 1991). Nearly all of these subprotoplasts, mostly cytoplasts, had deposited new cell walls detectable by Calcofluor staining within an hour after the start of regeneration in enriched W 5 medium (Kroh and Knuiman 1988). The rapid synthesis of new cell walls in these subprotoplasts probably reflects their isolation from rapidly growing pollen tubes and their culture in a nutrient-rich medium (Kroh and Knuiman 1988). Cell wall synthesis in Mougeotia cytoplasts differs signifi- cantly from the above examples because it occurs in a relatively nutrient-poor sorbitol and mineral salts medium.

The ability of Mougeotia cytoplasts to initiate cell wall synthesis and MT reorganisation, and to develop axiality (as exhibited in the formation of polar MT arrays) exemplifies the complexity of cellular activity that can occur in the absence of direct nuclear control in plant cytoplasts. This protoplasts/cytoplasts system may prove useful for investigating the molecular basis of protoplast axiality and regeneration, as well as providing further insights into cytoskeletal activity and cytoplasmic interactions independent of direct nuclear influence.

\section{Acknowledgements}

M.E.G. acknowledges the award of a postgraduate scholarship by the Australian National University. It is a pleasure for G.J.H. to acknowledge the encouragement and assistance of Dr. Robyn Overall.

\section{References}

Bajer AS, Molè-Bajer J (1982) Asters, poles, and transport properties within spindlelike microtubule arrays. Cold Spring Harbor Symp Quant Biol 46: 263-283

- (1986) Reorganization of microtubules in endosperm cells and cell fragments of the higher plant Haemanthus in vivo. $\mathbf{J}$ Cell Biol 102: 263-281

Bershadsky AD, Vasiliv JM (1988) Cytoskeleton. Plenum, New York Bilkey PC, Davey MR, Cocking EC (1982) Isolation, origin and properties of enucleate plant microplasts. Protoplasma 110: 147152

Bunn CL (1982) The influence of cytoplast-to-cell ratio on cybrid formation. In: Shay JW (ed) Techniques in somatic cell genetics. Plenum, New York, pp 189-201

Burridge K, Fath K, Kelly T, Nuckolls G, Turner C (1988) Focal adhesions: transmembrane junctions between the extracellular matrix and the cytoskeleton. Annu Rev Cell Biol 4: 487-525

Chang-Jie J, Sonobe S, Shibaoka H (1992) Assembly of microtubules in a cytoplasmic extract of tobacco BY-2 miniprotoplasts in the absence of microtubule-stabilizing agents. Plant Cell Physiol 33: 497-501

Cyr RJ (1991) Microtubule-associated proteins in higher plants. In: Lloyd CW (ed) The cytoskeletal basis of plant growth and form. Academic Press, London, pp 57-67

Galway ME, Hardham AR (1986) Microtubule reorganization, cell wall synthesis and establishment of the axis of elongation in regenerating protoplasts of the alga Mougeotia. Protoplasma 135: $130-143$

- - (1989) Oryzalin-induced microtubule disassembly and recovery in regenerating protoplasts of the alga Mougeotia. J Plant Physiol 135: 337-345

Gelfand VI, Glushankova NA, Ivanova OYu, Mittelman LA, Pletyushkina OYu, Vasiliev JM, Gelfand IM (1985) Polarization of cytoplasmic fragments microsurgically detached from mouse fibroblasts. Cell Biol Int Rep 9: 883-892 
Guha Roy S, Bhisey AN (1992) Cytoplasmic microtubule assembly is altered in cytoplasts. Cell Biol Int Rep 16: 269-281

Gunning BES, Hardham AR (1982) Microtubules. Annu Rev Plant Physiol 33: 651-698

Hughes J, McCully ME (1975) The use of an optical brightener in the study of plant structure. Stain Technol 50:319--329

Ingber DE (1993) Cellular tensegrity: defining new rules of biological design that govern the cytoskeleton. J Cell Sci 104: 613-627

Itoh T, Legge RL, Brown RM Jr (1986) The effects of selected inhibitors on cellulose microfibril assembly in Boergesenia forbesii (Chlorophyta) protoplasts. J Phycol 22: 224-233

Karsenti E, Kobayashi S, Mitchison T, Kirschner M (1984) Role of the centrosome in organizing the interphase microtubule array: properties of cytoplasts containing or lacking centrosomes. J Cell Biol 98: 1763-1776

Kennedy FGR, Hoshaw RW (1978) Culture study of reproductive cycles and systematics in Mougeotia transeaui (Chlorophyta). J Phycol 14: 445-450

Kopecká M, Gabriel M, Farkas V (1987) Some enucleated yeast protoplasts synthesize $\beta$ - $(1,3)$-D-glucan microfibrils of the cell wall. Naturwissenschaften 74: $389-391$

Kroh M, Knuiman B (1988) Development of subprotoplasts from in vitro-grown tobacco pollen tubes. Sex Plant Reprod 1: 103113

Maeda H, Ishida N (1967) Specificity of binding of hexopyranosyl polysaccharides with fluorescent brightener. J Biochem 62: 276278

Marchant HJ (1979) Microtubules, cell wall deposition and the determination of plant cell shape. Nature 278: 167-168

- Fowke LC (1977) Preparation, culture, and regeneration of protoplasts from filamentous green algae. Can J Bot 55: 3080-3086

- Hines ER (1979) The role of microtubules and cell-wall deposition in elongation of regenerating protoplasts of Mougeotia. Planta 146: $41-48$
Osborn M, Weber K (1982) Immunofluorescence and immunocytochemical procedures with affinity purified antibodies: tubulincontaining structures. In: Wilson L (ed) Methods in cell biology, vol 24, the cytoskeleton, part A. Academic Press, New York, pp $97-132$

Raghow R (1987) Regulation of messenger RNA turnover in eukaryotes. Trends Biochem Sci 12: 358-60

Reinert J, Binding H (1986) Protoplast fusion and early development of fusants. In: Reinert $\mathbf{J}$, Binding $\mathbf{H}$ (eds) Differentiation of protoplasts and of transformed plant cells. Springer, Berlin Heidelberg New York Tokyo, pp 37-66 [Hennig W, Nover L, Scheer $\mathrm{U}$ (eds) Problems in cell differentiation, vol 12]

Roberts K (1989) The plant extracellular matrix. Curr Opin Cell Biol 1: $1020-1027$

- (1990) Structures at the plant cell surface. Curr Opin Cell Biol 2: $920-928$

Rutten TLM, Derksen J (1990) Organization of actin filaments in regenerating and outgrowing subprotoplasts from pollen tubes of Nicotiana tabacum L. Planta 180: 471-479

- - (1992) Microtubules in pollen tube subprotoplasts: organization during protoplasts formation and protoplast outgrowth. Protoplasma 167: 231-237

- Kroh M, Knuiman B (1991) Cell-wall regeneration in pollentube subprotoplasts. Acta Bot Neerl 40: 211-216

Schweiger H-G, Berger S (1979) Nucleocytoplasmic interrelationships in Acetabularia and some other Dasycladaceae. Int Rev Cytol [Suppl 9]: pp 11-44

White RG, Hyde GJ, Overall RL (1990) Microtubule arrays in regenerating Mougeotia protoplasts may be oriented by electric fields. Protoplasma 158: 73-85

Williamson RE (1991) Orientation of cortical microtubules in interphase plant cells. Int Rev Cytol 129: 135-206 